Case Report

\title{
Antineutrophil Cytoplasmic Antibody Induction due to Infection: A Patient with Infective Endocarditis and Chronic Hepatitis C
}

\author{
Fareed B. Kamar ${ }^{1}$ and T. Lee-Ann Hawkins ${ }^{2}$ \\ ${ }^{1}$ Department of Medicine, University of Calgary, Calgary, AB, Canada T2N 4N1 \\ ${ }^{2}$ Department of Medicine, Division of General Internal Medicine, University of Calgary, Calgary, AB, Canada T2N 4N1
}

Correspondence should be addressed to Fareed B. Kamar; fbkamar@ucalgary.ca

Received 10 June 2015; Accepted 21 December 2015

Copyright ( 2016 F. B. Kamar and T. L.-A. Hawkins. This is an open access article distributed under the Creative Commons Attribution License, which permits unrestricted use, distribution, and reproduction in any medium, provided the original work is properly cited.

\begin{abstract}
While antineutrophil cytoplasmic antibody (ANCA) is often used as a diagnostic marker for certain vasculitides, ANCA induction in the setting of infection is much less common. In the case of infective endocarditis, patients may present with multisystem disturbances resembling an autoimmune process, cases that may be rendered even trickier to diagnose in the face of a positive ANCA. Though not always straightforward, distinguishing an infective from an inflammatory process is pivotal in order to guide appropriate therapy. We describe an encounter with a 43-year-old male with chronically untreated hepatitis $\mathrm{C}$ virus infection who featured ANCA positivity while hospitalized with acute bacterial endocarditis. His case serves as a reminder of two of the few infections known to uncommonly generate ANCA positivity. We also summarize previously reported cases of ANCA positivity in the context of endocarditis and hepatitis $\mathrm{C}$ infections.
\end{abstract}

\section{Introduction}

The antineutrophil cytoplasmic antibody (ANCA) class of immunoglobulins features the principal subtypes c-ANCA and p-ANCA, which are predominantly generated against the cytosolic antigens proteinase 3 (PR3) and myeloperoxidase (MPO), respectively [1]. The presence of these autoantibodies has been described in a variety of autoimmune conditions, such as small-vessel vasculitides, ulcerative colitis, primary sclerosing cholangitis, and autoimmune hepatitis $[2,3]$. Less frequently, ANCA induction can occur due to infections such as amebiasis, endocarditis, tuberculosis, malaria, human immunodeficiency virus infection, and hepatitis $C$ virus (HCV) infection [2, 4]. Because autoimmune and infectious diseases may present similarly, ANCA positivity must be carefully interpreted [5]. The following case describes a 43year-old male with chronically untreated $\mathrm{HCV}$ infection who was admitted to hospital with infective endocarditis and was found to be c-ANCA positive. We also summarize the literature concerning ANCA positivity in endocarditis and HCV infections.

\section{Clinical Vignette}

A 43-year-old male with a history of HCV infection (untreated since his diagnosis six years previously, with an RNA viral load of $1584 \mathrm{IU} / \mathrm{mL}$ on admission) and intravenous polysubstance use presented to a medical center with acute fever, dyspnea, and arthralgia. He was found to have purpura over his edematous lower extremities. His initial laboratory investigations featured an elevated white blood cell count of $16 \times 10^{9}$ cells per liter, elevated C-reactive protein of $183 \mathrm{mg} / \mathrm{L}$, urinalysis that was positive for hematuria, and blood cultures that were later positive for methicillin-sensitive Staphylococcus aureus. He did feature transient acute kidney injury soon after admission (peak serum creatinine $283 \mathrm{umol} / \mathrm{L}$ ). His serology was also positive for c-ANCA (anti-PR3), antinuclear antibody, and weakly positive for type III cryoglobulinemia. An echocardiogram revealed $1.1 \times 1.3 \mathrm{~cm}$ tricuspid vegetation involving the anterior and septal leaflets. A computerized tomography scan of his chest illustrated multiple bilateral septic pulmonary emboli, bilateral pleural effusions, and an anterior mediastinal abscess. A punch biopsy of a purpuric 
lesion on his right shin, performed one week into antibiotic therapy as his purpura was resolving (Figure 1(a)), revealed mild perivascular inflammation with focally extravasated erythrocytes and hemosiderin deposits consistent with a mild or resolving purpuric process (Figure 1(b)). The patient demonstrated clinical improvement during a six-week course of cefazolin.

\section{Discussion}

ANCA positivity may pose a diagnostic and therapeutic quandary in the face of patient presentation consistent with either a vasculitic or infectious process, particularly in the case of infective endocarditis [6].

A literature search of previously published cases concerning ANCA induction in infective endocarditis was performed via PubMed and Medline using the title and abstract entries "endocarditis" and "ANCA or antineutrophil cytoplasmic antibody," yielding 70 relevant cases [1,5-39]. A recent publication by Ying et al. (2014) describes 13 of these cases in addition to a literature review of several other ones [7]. We have expanded on this review through the addition of 26 other cases (Table 1).

A set of diagnostic aids between infective endocarditis and small-vessel vasculitis has been previously outlined (Table 2) [8]. One similarity, for example, is acute renal failure, the prevalence of which in bacterial endocarditis is $30 \%$ and is a significant predictor of mortality [40]. Glomerulonephritis in infective endocarditis is either pauci-immune, postinfective, or subendothelial membranoproliferative [41], the etiology of which can usually be discerned by obtaining a kidney biopsy [8].

Another ANCA-associated infection present in our reported patient is HCV infection. Previously published cases of ANCA induction due to hepatitis $\mathrm{C}$ infection are also summarized (Table 3) $[6,9,42-54]$. Our case report hence features two possible infections for c-ANCA induction, both of which likely also contributed to the patient's cryoglobulinemia. Because ANCA induction is more common in chronic infections [6], it argues for hepatitis $C$ as the cause of this patient's ANCA positivity as opposed to the more acute infection Staphylococcus aureus endocarditis [55]. Had his ANCA status been checked after endocarditis recovery, ANCA induction due to endocarditis as opposed to hepatitis $\mathrm{C}$ would have also been supported by a normalized or negative ANCA titer [11].

\section{Conclusion}

In light of its use in the diagnostic evaluation of vasculitis, a positive ANCA may allow for an infection to mislead a diagnostician down the path of autoimmune possibilities, particularly in the context of infective endocarditis. While clues may be drawn from clinical, laboratory, and radiological data to help differentiate infective endocarditis from vasculitis, obtaining blood cultures is of foremost importance. Making such a distinction will avoid the detrimental consequence of initiating immunosuppressive therapy against an infection masquerading as an inflammatory disease.
TABLE 1: Number of positive clinical and laboratory characteristics among all previously reported cases of ANCA-positive infective endocarditis* .

\begin{tabular}{|c|c|}
\hline Patient characteristic & $\begin{array}{c}\text { Proportion among } \\
70 \text { reported patient } \\
\text { cases }\end{array}$ \\
\hline Mean age (years) & 53.2 \\
\hline Male/female & $54 / 16$ \\
\hline Valve involvement & $56 / 70$ \\
\hline Aortic & 22 \\
\hline Mitral & 16 \\
\hline Left-sided not otherwise specified & 7 \\
\hline Aortic plus mitral & 6 \\
\hline Tricuspid & 5 \\
\hline Pulmonary & 1 \\
\hline Mitral plus pulmonary and tricuspid & 1 \\
\hline Ventricular septal defect & 1 \\
\hline \multicolumn{2}{|l|}{ Clinical features } \\
\hline Fever & 46 \\
\hline Anemia & 34 \\
\hline Splenomegaly & 19 \\
\hline Nephropathy (GN or AKI) & 43 \\
\hline Arthralgia & 17 \\
\hline Lower extremity edema & 23 \\
\hline Rash & 15 \\
\hline Purpura & 11 \\
\hline Cerebral infarction & 7 \\
\hline Finger clubbing & 4 \\
\hline \multicolumn{2}{|l|}{ Laboratory results } \\
\hline PR3 & 52 \\
\hline MPO & 8 \\
\hline $\mathrm{PR} 3+\mathrm{MPO}$ & 7 \\
\hline Hematuria & 49 \\
\hline Proteinuria & 14 \\
\hline \multicolumn{2}{|l|}{ Microbiology } \\
\hline Positive blood culture & $54 / 70$ \\
\hline \multicolumn{2}{|l|}{ Pathogen } \\
\hline Streptococcus spp. & 28 \\
\hline Enterococcus spp. & 7 \\
\hline Staphylococcus spp. & 10 \\
\hline Bartonella spp. & 9 \\
\hline Neisseria spp. & 1 \\
\hline Propionibacterium spp. & 1 \\
\hline Haemophilus spp. & 1 \\
\hline Gemella spp. & 1 \\
\hline Aggregatibacter spp. & 1 \\
\hline
\end{tabular}

GN: glomerulonephritis; AKI: acute kidney injury; PR3: proteinase 3; MPO: myeloperoxidase; spp.: species.

*This table, taken from Ying et al. (2014) with permission, expands the review from the original 44 patients to include 26 others [7]. 
TABLE 2: Diagnostic aids for differentiating between infectious endocarditis and small-vessel vasculitis* .

\begin{tabular}{ll}
\hline Similarities $^{\mathrm{a}}$ & Differences $^{\mathrm{b}}$ \\
\hline (i) Presentation with & (i) Splenomegaly \\
constitutional symptoms & (ii) Thrombocytopenia \\
(ii) Pyrexia & (iii) Hypocomplementemia \\
(iii) Active urinary & (iv) Immune complexes \\
sediment & (v) Other positive autoantibodies \\
(iv) Skin involvement & (vi) Low titer ANCA/ELISA negative \\
(v) Decreased GFR & (vii) Other organ involvement \\
(vi) Increased & inflammatory marker levels
\end{tabular}

ANCA: antineutrophil cytoplasmic antibody; ELISA: enzyme-linked immunosorbent assay; GFR: glomerular filtration rate.

${ }^{\mathrm{a}}$ Features seen in both conditions.

${ }^{\mathrm{b}}$ Features seen predominantly in infectious endocarditis.

${ }^{*}$ This table was taken from Forbes et al. (2012) with permission [8].

TABLE 3: Summary of previously published ANCA-positive hepatitis C infection cases.

\begin{tabular}{|c|c|c|c|}
\hline Paper & $\begin{array}{l}\text { Age (years), } \\
\text { sex }\end{array}$ & ANCA & Miscellaneous features \\
\hline \multirow{3}{*}{$\begin{array}{l}\text { Bonaci-Nikolic et } \\
\text { al., } 2010[6]\end{array}$} & $63, \mathrm{~F}$ & MPO & - \\
\hline & $51, \mathrm{~F}$ & MPO & - \\
\hline & $24, \mathrm{~F}$ & MPO & - \\
\hline $\begin{array}{l}\text { Cojocaru et al., } 2007 \\
{[42]}\end{array}$ & Mean 75 & $21 \mathrm{PR} 3$ & Concomitant ischemic stroke \\
\hline $\begin{array}{l}\text { Cojocaru et al., } 2006 \\
{[43]}\end{array}$ & $?$ & $?$ & - \\
\hline $\begin{array}{l}\text { Gatselis et al., } 2006 \\
{[44]}\end{array}$ & $?$ & $\begin{array}{l}65 \text { c-ANCA, } 4 \text { p-ANCA (though all negative } \\
\text { for PR3 and MPO) }\end{array}$ & \\
\hline \multirow{5}{*}{$\begin{array}{l}\text { Lamprecht et al., } \\
2003 \text { [9] }\end{array}$} & \multirow{5}{*}{$?$} & 6 bactericidal/permeability-increasing proteins & \multirow{3}{*}{ Mixed cryoglobulinemia } \\
\hline & & 4 cathepsin $\mathrm{G}$ proteins & \\
\hline & & 1 unknown antigen (c-ANCA) & \\
\hline & & 2 bactericidal/permeability-increasing proteins & \multirow{2}{*}{ No cryoglobulinemia } \\
\hline & & Four patients: cathepsin G & \\
\hline $\begin{array}{l}\text { Zandman-Goddard } \\
\text { et al., } 2003 \text { [45] }\end{array}$ & $34, \mathrm{M}$ & c-ANCA and p-ANCA & Complicated by transverse myelitis \\
\hline $\begin{array}{l}\text { Tajima et al., } 2002 \\
\text { [46] }\end{array}$ & $66, \mathrm{~F}$ & p-ANCA & Complicated by pachymeningitis \\
\hline Wu et al., 2002 [47] & $?$ & $\begin{array}{l}253 \text { PR3, } \\
25 \text { PR3 and MPO }\end{array}$ & $\begin{array}{l}\text { Higher proportion of ANCA-positive compared } \\
\text { to ANCA-negative patients with high alanine } \\
\text { aminotransferase, high alpha-fetoprotein, skin } \\
\text { disease, cirrhosis, and anemia }\end{array}$ \\
\hline $\begin{array}{l}\text { Agarwal et al., } 2001 \\
\text { [48] }\end{array}$ & $?$ & 5 p-ANCA & - \\
\hline Igaki et al., 2000 [49] & $60, \mathrm{~F}$ & MPO & Glomerulonephritis, cryoglobulinemia \\
\hline $\begin{array}{l}\text { Lamprecht et al., } \\
1998 \text { [50] }\end{array}$ & $60, \mathrm{~F}$ & c-ANCA & Type II cryoglobulinemia \\
\hline Ohira et al., 1998 [51] & $?$ & 12 c-ANCA or p-ANCA & - \\
\hline $\begin{array}{l}\text { Kallinowski et al., } \\
1997[52]\end{array}$ & $?$ & 5 ANCA & - \\
\hline Papi et al., 1997 [53] & $63, \mathrm{~F}$ & MPO & $\begin{array}{l}\text { Mixed type II cryoglobulinemia, leukocytoclastic } \\
\text { vasculitis on skin biopsy }\end{array}$ \\
\hline $\begin{array}{l}\text { Dalekos and } \\
\text { Tsianos, } 1994[54]\end{array}$ & $?$ & 3 ANCA & - \\
\hline
\end{tabular}

F: female; ANCA: antineutrophil cytoplasmic antibody; MPO: myeloperoxidase; PR3: proteinase 3. 


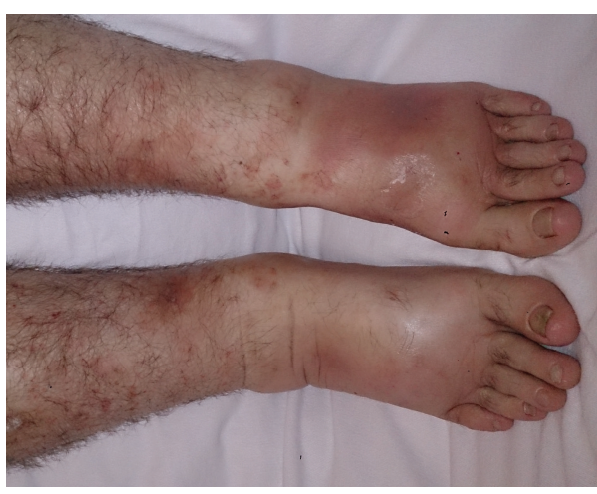

(a)

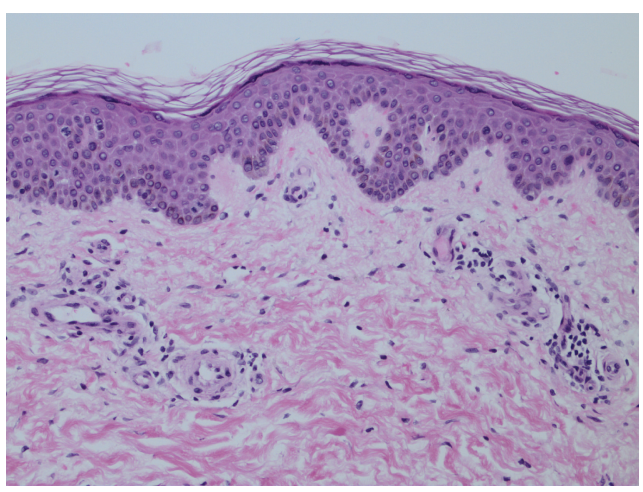

(b)

FIGURE 1: (a) Photograph of the patient's resolving purpura involving his legs one week into antibiotic therapy. (b) Corresponding hematoxylin and eosin-stained histopathology at 20x magnification of a punch biopsy of one of the lesions on his leg, showing mild perivascular inflammation with focally extravasated erythrocytes consistent with a resolving purpuric process. No leukocytoclastic vasculitis was seen.

\section{Competing Interests}

There are no competing interests to disclose between both authors.

\section{Acknowledgments}

The authors recognize the pathologist Dr. Karen Naert (Foothills Medical Centre, Calgary, Alberta, Canada) as a contributor to this paper for her analysis of the pathology specimen and for supplying the histology image (Figure 1(b)).

\section{References}

[1] B. Hellmich, M. Ehren, M. Lindstaedt, M. Meyer, M. Pfohl, and H. Schatz, "Anti-MPO-ANCA-positive microscopic polyangiitis following subacute bacterial endocarditis," Clinical Rheumatology, vol. 20, no. 6, pp. 441-443, 2001.

[2] D. Vassilopoulos and G. S. Hoffman, "Clinical utility of testing for anti-neutrophil cytoplasmic antibodies," Clinical and Diagnostic Laboratory Immunology, vol. 6, no. 5, pp. 645-651, 1999.

[3] J. U. Holle and W. L. Gross, "ANCA-associated vasculitides: pathogenetic aspects and current evidence-based therapy," Journal of Autoimmunity, vol. 32, no. 3-4, pp. 163-171, 2009.

[4] E. Csernok, P. Lamprecht, and W. L. Gross, "Clinical and immunological features of drug-induced and infection-induced proteinase 3-antineutrophil cytoplasmic antibodies and myeloperoxidase- antineutrophil cytoplasmic antibodies and vasculitis," Current Opinion in Rheumatology, vol. 22, no. 1, pp. 4348, 2010.

[5] I. Veerappan, E. N. Prabitha, A. Abraham, S. Theodore, and G. Abraham, "Double ANCA-positive vasculitis in a patient with infective endocarditis," Indian Journal of Nephrology, vol. 22, no. 6, pp. 469-472, 2012.

[6] B. Bonaci-Nikolic, S. Andrejevic, M. Pavlovic, Z. Dimcic, B. Ivanovic, and M. Nikolic, "Prolonged infections associated with antineutrophil cytoplasmic antibodies specific to proteinase 3 and myeloperoxidase: diagnostic and therapeutic challenge," Clinical Rheumatology, vol. 29, no. 8, pp. 893-904, 2010.

[7] C.-M. Ying, D.-T. Yao, H.-H. Ding, and C.-D. Yang, "Infective endocarditis with antineutrophil cytoplasmic antibody: report of 13 cases and literature review," PLoS ONE, vol. 9, no. 2, Article ID e89777, 2014.

[8] S. H. Forbes, S. C. Robert, J. E. Martin, and R. Rajakariar, "Acute kidney injury with hematuria, a positive ANCA test, and low levels of complement," American Journal of Kidney Diseases, vol. 59, no. 1, pp. 28-31, 2012.

[9] P. Lamprecht, O. Gutzeit, E. Csernok et al., "Prevalence of ANCA in mixed cryoglobulinemia and chronic hepatitis C virus infection," Clinical and Experimental Rheumatology, vol. 21, no. 6, pp. S89-S94, 2003.

[10] A. Bauer, W. J. Jabs, S. Süfke, M. Maass, and B. Kreft, "Vasculitic purpura with antineutrophil cytoplasmic antibodypositive acute renal failure in a patient with Streptococcus bovis and Neisseria subflava bacteremia and subacute endocarditis," Clinical Nephrology, vol. 62, no. 2, pp. 144-148, 2004.

[11] J. A. Chirinos, V. F. Corrales-Medina, S. Garcia, D. M. Lichtstein, A. L. Bisno, and S. Chakko, "Endocarditis associated with antineutrophil cytoplasmic antibodies: a case report and review of the literature," Clinical Rheumatology, vol. 26, no. 4, pp. 590595, 2007.

[12] H. K. Choi, P. Lamprecht, J. L. Niles, W. L. Gross, and P. A. Merkel, "Subacute bacterial endocarditis with positive cytoplasmic antineutrophil cytoplasmic antibodies and antiproteinase 3 antibodies," Arthritis and Rheumatism, vol. 43, no. 1, pp. 226-231, 2000.

[13] A. de Corla-Souza and B. A. Cunha, "Streptococcal viridans subacute bacterial endocarditis associated with antineutrophil cytoplasmic autoantibodies (ANCA)," Heart and Lung, vol. 32, no. 2, pp. 140-143, 2003.

[14] H. Fukasawa, M. Hayashi, N. Kinoshita et al., "Rapidly progressive glomerulonephritis associated with PR3-ANCA positive subacute bacterial endocarditis," Internal Medicine, vol. 51, no. 18, pp. 2587-2590, 2012.

[15] M. Fukuda, M. Motokawa, T. Usami et al., "PR3-ANCA-positive crescentic necrotizing glomerulonephritis accompanied by isolated pulmonic valve infective endocarditis, with reference to previous reports of renal pathology," Clinical Nephrology, vol. 66, no. 3, pp. 202-209, 2006.

[16] G. C. Ghosh, B. Sharma, B. Katageri, and M. Bhardwaj, "ANCA positivity in a patient with infective endocarditis-associated glomerulonephritis: a diagnostic dilemma," Yale Journal of Biology and Medicine, vol. 87, no. 3, pp. 373-377, 2014. 
[17] W. Hanf, J. E. Serre, J. H. Salmon et al., "Glomerulonephrite rapidement progressive a ANCA revelant une endocardite infectieuse subaigue (Rapidly progressive ANCA positive glomerulonephritis as the presenting feature of infectious endocarditis)," La Revue de Médecine Interne, vol. 32, no. 12, pp. el16el18, 2011.

[18] T. Haseyama, H. Imai, A. Komatsuda et al., "Proteinase3-antineutrophil cytoplasmic antibody (PR3-ANCA) positive crescentic glomerulonephritis in a patient with Down's syndrome and infectious endocarditis," Nephrology Dialysis Transplantation, vol. 13, no. 8, pp. 2142-2146, 1998.

[19] T. Hirunagi, H. Kawanishi, N. Mitsuma, Y. Goto, and K. Mano, "Aggregatibacter segnis endocarditis mimicking antineutrophil cytoplasmic antibody-associated vasculitis presenting with cerebral hemorrhage: a case report," Rinsho Shinkeigaku, vol. 55, no. 8, pp. 589-592, 2015.

[20] A. H. Holmes, T. C. Greenough, G. J. Balady et al., "Bartonella henselae endocarditis in an immunocompetent adult," Clinical Infectious Diseases, vol. 21, no. 4, pp. 1004-1007, 1995.

[21] Y. Kawamorita, Y. Fujigaki, A. Imase et al., "Successful treatment of infectious endocarditis associated glomerulonephritis mimicking c3 glomerulonephritis in a case with no previous cardiac disease," Case Reports in Nephrology, vol. 2014, Article ID 569047, 6 pages, 2014.

[22] N. Kishimoto, Y. Mori, H. Yamahara et al., "Cytoplasmic antineutrophil cytoplasmic antibody positive pauci-immune glomerulonephritis associated with infectious endocarditis," Clinical Nephrology, vol. 66, no. 6, pp. 447-454, 2006.

[23] K. N. Konstantinov, A. A. Harris, M. F. Hartshorne, and A. H. Tzamaloukas, "Symptomatic antineutrophil cytoplasmic antibody-positive disease complicating subacute bacterial endocarditis: to treat or not to treat?" Case Reports in Nephrology and Urology, vol. 2, no. 2, pp. 25-32, 2012.

[24] A. Mahr, F. Batteux, S. Tubiana et al., "Brief report: prevalence of antineutrophil cytoplasmic antibodies in infective endocarditis," Arthritis and Rheumatology, vol. 66, no. 6, pp. 1672-1677, 2014.

[25] S. P. McAdoo, C. Densem, A. Salama, and C. D. Pusey, "Bacterial endocarditis associated with proteinase 3 anti-neutrophil cytoplasm antibody," NDT Plus, vol. 4, no. 3, pp. 208-210, 2011.

[26] K. Osafune, H. Takeoka, H. Kanamori et al., "Crescentic glomerulonephritis associated with infective endocarditis: renal recovery after immediate surgical intervention," Clinical and Experimental Nephrology, vol. 4, no. 4, pp. 329-334, 2000.

[27] H. Peng, W.-F. Chen, C. Wu et al., "Culture-negative subacute bacterial endocarditis masquerades as granulomatosis with polyangiitis (Wegener's granulomatosis) involving both the kidney and lung," BMC Nephrology, vol. 13, article 174, 2012.

[28] A. M. Riding and D. P. D'Cruz, "A case of mistaken identity: subacute bacterial endocarditis associated with p-antineutrophil cytoplasmic antibody," BMJ Case Reports, 2010.

[29] C. Salvado, A. Mekinian, P. Rouvier, P. Poignard, I. Pham, and O. Fain, "Rapidly progressive crescentic glomerulonephritis and aneurism with antineutrophil cytoplasmic antibody: Bartonella henselae endocarditis," Presse Medicale, vol. 42, no. 6, part 1, pp. 1060-1061, 2013.

[30] K. Satake, I. Ohsawa, N. Kobayashi et al., "Three cases of PR3ANCA positive subacute endocarditis caused by attenuated bacteria (Propionibacterium, Gemella, and Bartonella) complicated with kidney injury," Modern Rheumatology, vol. 21, no. 5, pp. 536-541, 2011.
[31] S. H. Shah, C. Grahame-Clarke, and C. N. Ross, "Touch not the cat bot a glove* : ANCA-positive pauci-immune necrotizing glomerulonephritis secondary to Bartonella henselae," Clinical Kidney Journal, vol. 7, no. 2, pp. 179-181, 2014.

[32] A. Soto, C. Jorgensen, F. Oksman, L.-H. Noel, and J. Sany, "Endocarditis associated with ANCA," Clinical and Experimental Rheumatology, vol. 12, no. 2, pp. 203-204, 1994.

[33] J. F. Subra, C. Michelet, J. Laporte et al., "The presence of cytoplasmic antineutrophil cytoplasmic antibodies (C-ANCA) in the course of subacute bacterial endocarditis with glomerular involvement, coincidence or association?" Clinical Nephrology, vol. 49, no. 1, pp. 15-18, 1998.

[34] H. Sugiyama, M. Sahara, Y. Imai et al., "Infective endocarditis by Bartonella quintana masquerading as antineutrophil cytoplasmic antibody-associated small vessel vasculitis," Cardiology, vol. 114, no. 3, pp. 208-211, 2009.

[35] A. M. Tiliakos and N. A. Tiliakos, "Dual ANCA positivity in subacute bacterial endocarditis," Journal of Clinical Rheumatology, vol. 14, no. 1, pp. 38-40, 2008.

[36] M. Uh, I. A. McCormick, and J. T. Kelsall, "Positive cytoplasmic antineutrophil cytoplasmic antigen with PR3 specificity glomerulonephritis in a patient with subacute bacterial endocarditis," Journal of Rheumatology, vol. 38, no. 7, pp. 1527-1528, 2011.

[37] H. R. Vikram, A. K. Bacani, P. A. DeValeria, S. A. Cunningham, and F. R. Cockerill III, "Bivalvular Bartonella henselae prosthetic valve endocarditis," Journal of Clinical Microbiology, vol. 45, no. 12, pp. 4081-4084, 2007.

[38] J. Wagner, K. Andrassy, and E. Ritz, "Is vasculitis in subacute bacterial endocarditis associated with ANCA?" The Lancet, vol. 337, no. 8744, pp. 799-800, 1991.

[39] J. I. Zeledon, R. L. McKelvey, K. S. Servilla et al., "Glomerulonephritis causing acute renal failure during the course of bacterial infections," International Urology and Nephrology, vol. 40, no. 2, pp. 461-470, 2008.

[40] J. P. Gagliardi, R. E. Nettles, D. E. McCarty, L. L. Sanders, G. R. Corey, and D. J. Sexton, "Native valve infective endocarditis in elderly and younger adult patients: comparison of clinical features and outcomes with use of the Duke criteria and the Duke Endocarditis Database," Clinical Infectious Diseases, vol. 26, no. 5, pp. 1165-1168, 1998.

[41] A. Majumdar, S. Chowdhary, M. A. S. Ferreira et al., "Renal pathological findings in infective endocarditis," Nephrology Dialysis Transplantation, vol. 15, no. 11, pp. 1782-1787, 2000.

[42] I. M. Cojocaru, M. Cojocaru, and C. Burcin, "Ischemic stroke accompanied by anti-PR3 antibody-related cerebral vasculitis and hepatitis C virus infection," Romanian Journal of Internal Medicine, vol. 45, no. 1, pp. 47-50, 2007.

[43] M. Cojocaru, I. M. Cojocaru, and S. A. Iacob, "Prevalence of anti-neutrophil cytoplasmic antibodies in patients with chronic hepatitis C infection associated mixed cryoglobulinemia," Romanian Journal of Internal Medicine, vol. 44, no. 4, pp. 427431, 2006.

[44] N. K. Gatselis, S. P. Georgiadou, G. K. Koukoulis et al., “Clinical significance of organ- and non-organ-specific autoantibodies on the response to anti-viral treatment of patients with chronic hepatitis C," Alimentary Pharmacology and Therapeutics, vol. 24, no. 11-12, pp. 1563-1573, 2006.

[45] G. Zandman-Goddard, Y. Levy, P. Weiss, Y. Shoenfeld, and P. Langevitz, "Transverse myelitis associated with chronic hepatitis C," Clinical and Experimental Rheumatology, vol. 21, no. 1, pp. 111-113, 2003. 
[46] Y. Tajima, Y. Miyazaki, K. Sudoh, and A. Matumoto, "Intracranial hypertrophic pachymeningitis with high perinuclear antineutrophil cytoplasmic antibody (p-ANCA) occurred in a patient on hemodialysis," Clinical Neurology, vol. 42, no. 3, pp. 243-246, 2002.

[47] Y.-Y. Wu, T.-C. Hsu, T.-Y. Chen et al., "Proteinase 3 and dihydrolipoamide dehydrogenase (E3) are major autoantigens in hepatitis C virus (HCV) infection," Clinical and Experimental Immunology, vol. 128, no. 2, pp. 347-352, 2002.

[48] N. Agarwal, R. Handa, S. K. Acharya, J. P. Wali, A. K. Dinda, and P. Aggarwal, "A study of autoimmune markers in hepatitis C infection," Indian Journal of Medical Research, vol. 113, pp. 170174, 2001.

[49] N. Igaki, M. Nakaji, R. Moriguchi, H. Akiyama, F. Tamada, and T. Goto, "A case of hepatitis $\mathrm{C}$ virus-associated glomerulonephropathy presenting with MPO-ANCA-positive rapidly progressive glomerulonephritis," Japanese Journal of Nephrology, vol. 42, no. 4, pp. 353-358, 2000.

[50] P. Lamprecht, W. H. Schmitt, and W. L. Gross, "Mixed cryoglobulinaemia, glomerulonephritis, and ANCA: essential cryoglobulinaemic vasculitis or ANCA-associated vasculitis?" Nephrology Dialysis Transplantation, vol. 13, no. 1, pp. 213-221, 1998.

[51] H. Ohira, J. Tojo, J. Shinzawa et al., "Antineutrophil cytoplasmic antibody in patients with antinuclear antibody-positive chronic hepatitis C," Fukushima Journal of Medical Science, vol. 44, no. 2, pp. 83-92, 1998.

[52] B. Kallinowski, S. Seipp, S. Fatehi et al., "Significance of hepatitis $\mathrm{B}$, hepatitis $\mathrm{C}$ and GBV-C in ANCA-Positive hemodialysis patients," Nephron, vol. 77, no. 3, pp. 357-358, 1997.

[53] M. Papi, B. Didona, O. De Pita, M. Gantcheva, and L. M. Chinni, "Chronic hepatitis $\mathrm{C}$ virus infection, mixed cryoglobulinaemia, leukocytoclastic vasculitis and antineutrophil cytoplasmic antibodies," Lupus, vol. 6, no. 9, pp. 737-738, 1997.

[54] G. N. Dalekos and E. V. Tsianos, "Anti-neutrophil antibodies in chronic viral hepatitis," Journal of Hepatology, vol. 20, no. 4, p. $561,1994$.

[55] M. L. Fernandez Guerrero, J. J. Gonzalez Lopez, A. Goyenechea, J. Fraile, and M. de Gó rgolas, "Endocarditis caused by Staphylococcus aureus: a reappraisal of the epidemiologic, clinical, and pathologic manifestations with analysis of factors determining outcome," Medicine, vol. 88, no. 1, pp. 1-22, 2009. 


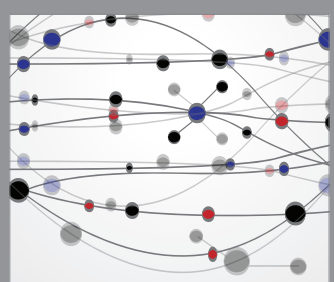

The Scientific World Journal
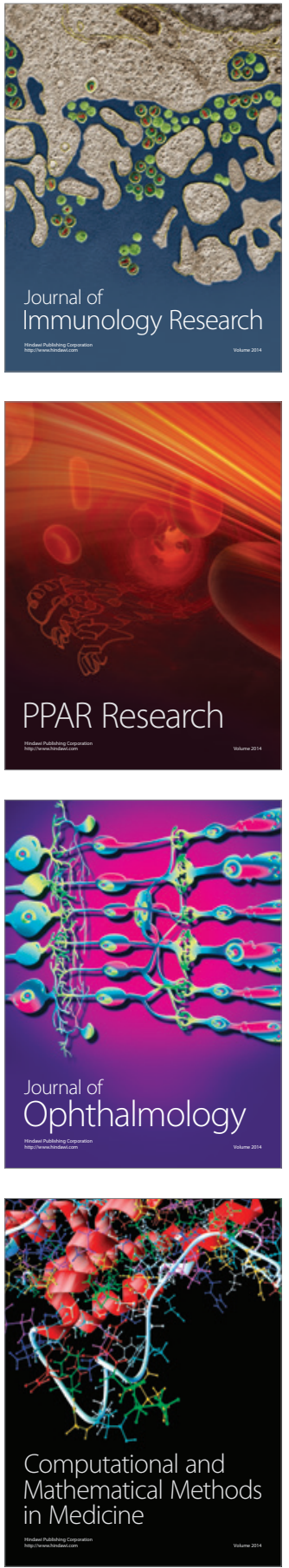

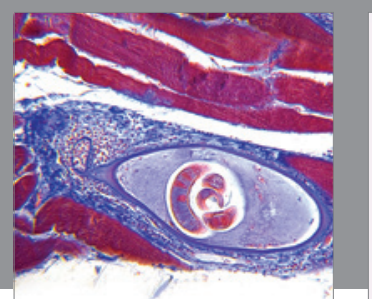

Gastroenterology Research and Practice

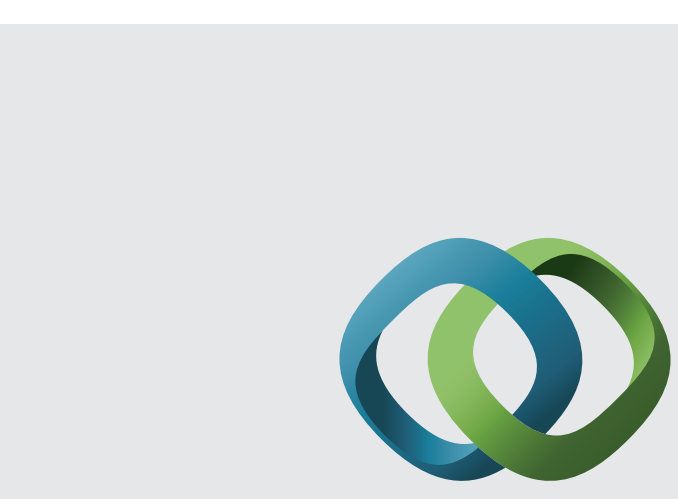

\section{Hindawi}

Submit your manuscripts at

http://www.hindawi.com
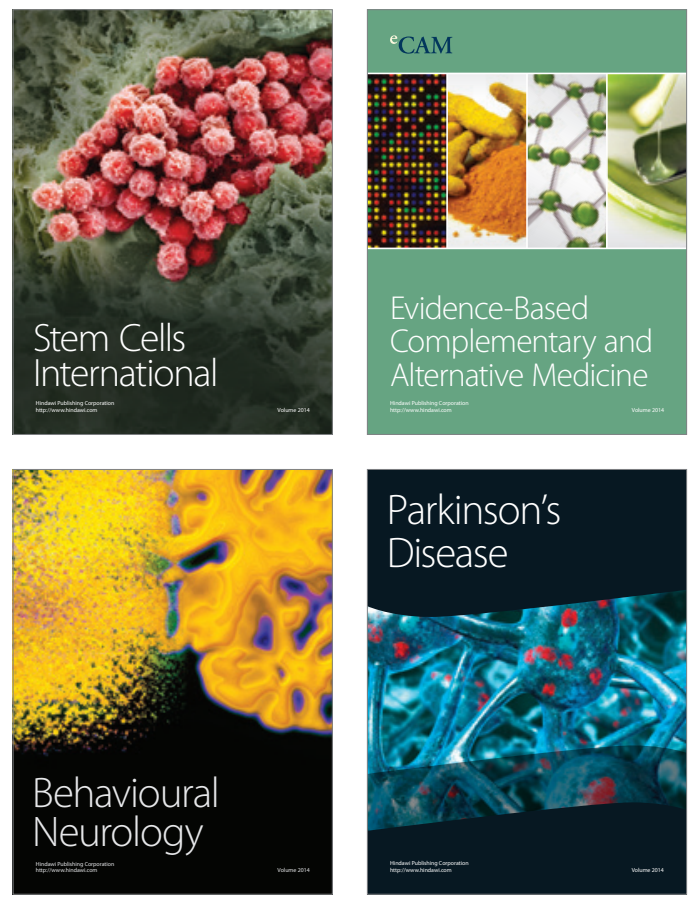
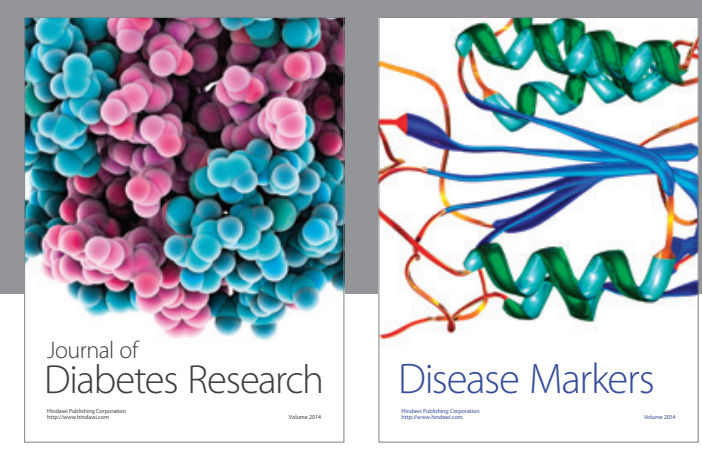

Disease Markers
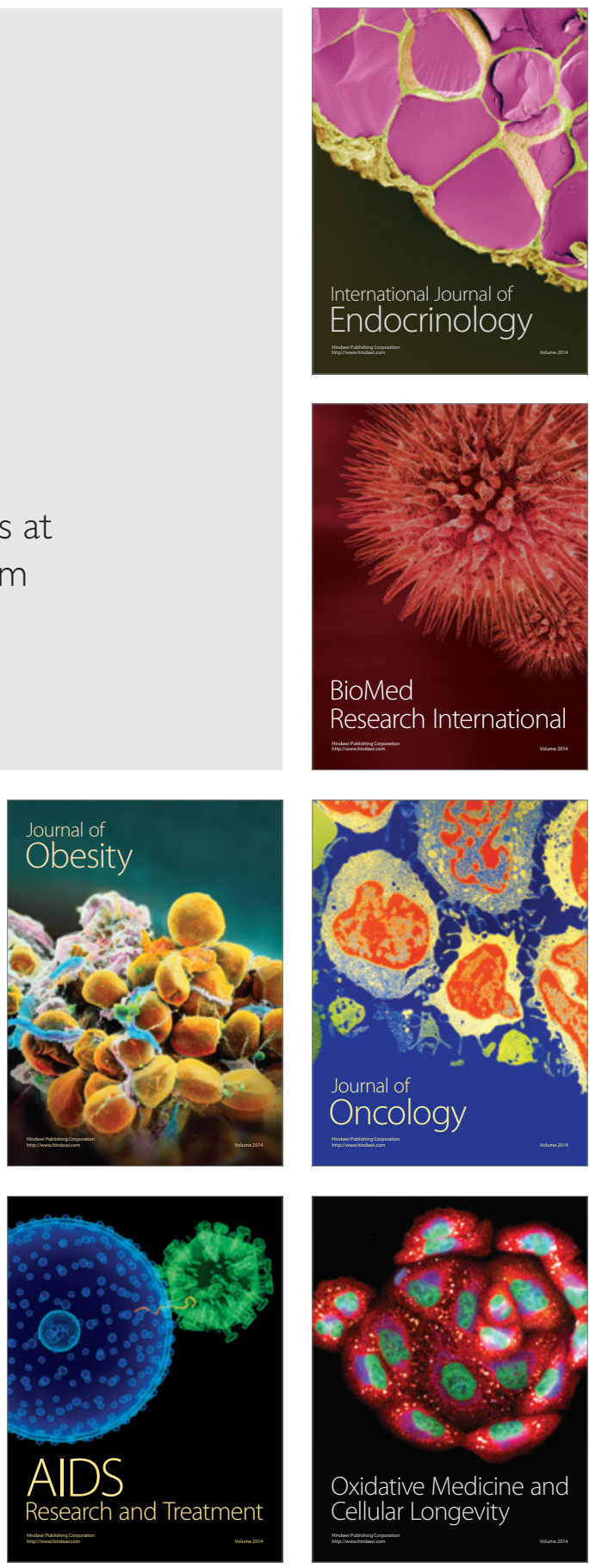